\title{
The Research on Soldier's Personalized Learning Based on improved Ant Colony Algorithm
}

\author{
Dong Li \& Huqiang Wang \\ Department of Information Engineer, Academy of Armored Forces Engineering
}

Keywords: Improved ant colony algorithm; Soldier's occupational skill education; personalized learning; optimal path.

\begin{abstract}
Confronting the situation of uneven educational background, knowledge comprehension and master speed of soldiers, stereotype education system of soldier's occupational skill no longer adapts the demand of network era development and informational military construction. The paper puts forward to the tactic of personalized learning of soldiers after analyzing the existing problems in soldier's occupational skill education and the shortcomings of traditional ant colony algorithm, the tactic which makes use of Max-Min ant system just up-dates the pheromones of the most optimal path the ant has went through, and the pheromones of each path are strictly limited. The algorithm will build related mathematical model and optimal strategy to find a personalized learning scheme or path that adapts to themselves according to the cognitive ability, self-learning style and knowledge level of soldiers. Then it will be provided to the soldiers so as to reach the purpose of improving their learning efficiency. The experiment result shows that the improved ant colony algorithm owns higher convergence, and the degree of coincidence between personalized learning path and the soldier's demand is greatly improved.
\end{abstract}

\section{Introduction}

Currently, soldier's occupational skill appraisal of armored equipment support consists of two parts: the theory test and the practical operation section. According to the analysis of appraisal results, most of the soldiers failed to pass the exam, a main reason is that they did not pass the theory test. In response to the situation that the pass rate of theory test is less than $60 \%$, while the pass rate of practical operation is as high as $90 \%$, this will inevitably lead to a situation that many talented people have to leave the troops because of their failure of theory test although their operation skill is great. Thus, how to improve the soldier's theoretical knowledge is significant not only to the soldier's comprehensive development, but also the military talented person construction. In order to satisfy the soldier's occupational skill education of different people, different levels and different specialties, the paper puts forward to the tactic of personalized learning based on improved ant colony algorithm, the improved algorithm takes soldier's learning style, knowledge master levels and whether it matches the learning resources into account, it further optimizes the update rules of pheromone, as a result, it can intelligently provide a most optimal learning path for the soldiers.

\section{Build model}

\subsection{Personalized feature extraction}

Soldier's feature description. Soldier's feature is divided into two parts: the learning style and knowledge master level, these also meet the unique learning way of everyone, as the following figure 1:

(1) Polymeric. These kinds of person are talented at discovering theoretical value, which can solve practical problems effectively; they are keen to the learning of text objects.

(2) Divergent. These kinds of person are talented at surveying the particular situation, they are keen to the learning objects contain charts, tables and animations.

(3)Assimilative. These kinds of person are interested in abstract concept, which needs interpretive explanation; they are keen to audios and videos. 
(4)Modulating. These kinds of person own favorable manipulative ability, they prefer challenging plans, and learn from experiences.

The same learner may own multiple learning styles; different learners also have different keen degrees on the same learning style. Thus, using the vector $s=\left(s_{1}, s_{2}, s_{3}, s_{4}\right)$ to describe the learner's style tendency $s_{1}, s_{2}, S_{3}, S_{4}$ stand for the tendency degree of the four learning styles, and $0 \leq s_{i} \leq 1(i=1,2,3,4), s_{1}+s_{2}+s_{3}+s_{4}=1$.

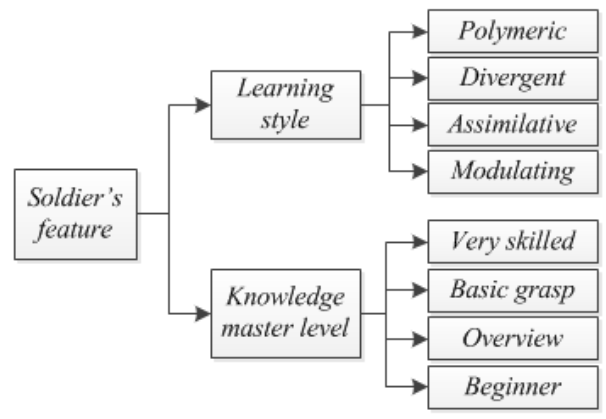

Figure.1 the classification of soldier's feature

Learning resource's description. Learning resources mainly involve knowledge expression and difficulty degree, as the Fig.2 shows. Generally, one learning resource contains multiple knowledge expression ways. Here, the vector $c=\left(c_{1}, c_{2}, c_{3}, c_{4}\right)$ is used to describe the proportion of every expression way, and $0 \leq c_{i} \leq 1(i=1,2,3,4)$,

$c_{1}+c_{2}+c_{3}+c_{4}=1$.

Also, the difficulty degree is obtained based on the span and depth of knowledge expression ways, it is set as $d(0 \leq d \leq 1)$. If $d$ approaches 1 , it means that the difficulty degree is easy, and it suits beginner, otherwise, it suits experts.

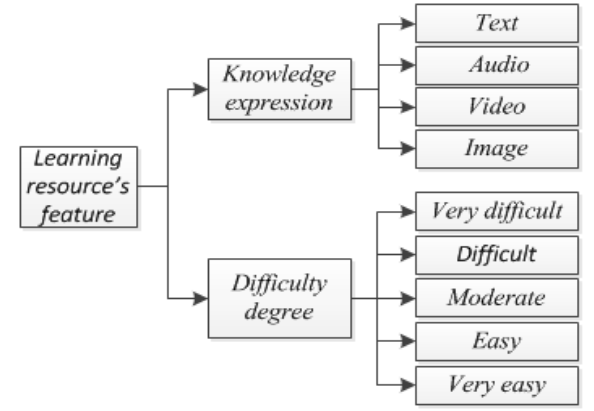

Figure.2 the classification of learning resource's feature

\subsection{Adjacent users recognition}

The choose of learning path usually draws support from two aspects:

What learning methods do the adjacent users who are similar to us in all aspects fond of, mainly reflects in knowledge master levels and learning styles.

Set $L=\left\{L_{1}, L_{2}, \ldots, L_{\mathrm{m}}\right\}$, it is a set consists of m learners, thus, the similar adjacent users whose knowledge master level are close to the target user. The formula is as following:

$L_{0, \text { similar }}^{k \text {-level }}=\left\{L_{j}|| l_{j}-l_{0} \mid \leq \gamma_{1}\right.$, and $\left.L_{j} \in L\right\}$

In the formula above, $L_{0}$ is the target user, $l_{0}$ is his knowledge master level, $\gamma_{1}$ and is the adjacent threshold of knowledge master level.

Thus, the adjacent users who not only satisfy the close of knowledge master level but also meet the close of learning style can be expressed like this:

$$
L_{0, \text { similar }}=\left\{L_{k}|| s^{0}-s^{k} \mid \leq \gamma_{2} \text {, and } L_{k} \in L_{0 \text {, similar }}^{k \text {-leve }}\right\}
$$

In the formula above, $s^{0}=\left(s_{1}^{0}, s_{2}^{0}, s_{3}^{0}, s_{4}^{0}\right)$ is the learning style of target user, $s^{k}=\left(s_{1}^{k}, s_{2}^{k}, s_{3}^{k}, s_{4}^{k}\right)$ is the learning style of k-th learner, $\left|s^{0}-s^{k}\right|$ is Euclidean distance, $\gamma_{2}$ and is adjacent threshold. 
The match degree between learning resources and knowledge master level. Whether the soldier's knowledge master level is coincide with the difficulty degree of learning resources, and their match degree is as the following formula:

$$
\eta_{L_{0}, o_{j}}^{k-\text { level }}=1-\left|l_{0}-d_{j}\right|
$$

In the formula above, $d_{j}$ is difficulty degree of learning resource $o_{j}$.

Whether the soldier's learning style is coincide with the knowledge expression ways, and their match degree is as the following formula:

$$
\eta_{L_{0}, o_{j}}^{l-s y l e}=1-\left|s^{0}-c^{j}\right|
$$

In the formula above, $c^{j}=\left(c_{1}^{j}, c_{2}^{j}, c_{3}^{j}, c_{4}^{j}\right)$ is the eigenvectors of knowledge expression way of $o_{j}$.

\section{Ant colony algorithm}

\subsection{The basic principle of ant colony algorithm}

Ant colony algorithm is put forward based on simulating the forage of natural ants, it is a complex group behavior derived from a series of simple individual behaviors. According to numerous studies, we find that the ants transmit information through a kind of substance called pheromones; they also secrete this kind of hormone during the process of movement and guide the next movement direction by perceiving the presence of this substance and its strength. Generally, the ants will move to the direction of higher pheromone strength, that is, while there are more ants in the path, more pheromone will be remained, and then the probability the path be chosen will be much more higher, this is a great help for the ants to find food quickly, this is seen as a kind of positive feedback mechanism of information.

\subsection{The choose of learning path by ant colony algorithm}

According to ant colony algorithm, the initial node of ant $\mathrm{k}$ is $\mathrm{i}$, at the time of $\mathrm{t}$, the proportion that the ant chooses the path $i \rightarrow j_{\text {is: }}$

$$
p_{i j}^{k}(t)= \begin{cases}\frac{\left[\eta_{i j}(t)\right]^{\alpha} \times\left[\tau_{i j}(t)\right]^{\beta}}{\sum\left(\left[\eta_{i j}(t)\right]^{\alpha} \times\left[\tau_{i j}(t)\right]^{\beta}\right)}, & j \in J(i) \\ 0, & j \notin J(i)\end{cases}
$$

In the formula above, $\eta_{i j}(t)$ is the heuristic information from $\mathrm{i}$ to $\mathrm{j}$, the heuristic information is gained according to concrete questions, for example, it can be the distance from $\mathrm{i}$ to $\mathrm{j}$ or price from $\mathrm{i}$ to j. $\tau_{i j}(t)$ is the pheromone concentration in the path of $i \rightarrow j$ at the time of $t ; J(i)$ is a path set the ant $\mathrm{k}$ will choose, $\alpha$ is the parameter of adjusting $\eta_{i j}(t), \beta$ is the parameter of adjusting $\tau_{i j}(t)$.

In addition, the volatile factor $\rho$ is introduced so as to eliminate the influence of initial state. Suppose there are $\mathrm{m}$ ants that get through the path of $i \rightarrow j$ at time t, thus, the update formula of pheromone is as following:

$$
\tau_{i j}(\mathrm{t}+1)=(1-\rho) \times \tau_{i j}(\mathrm{t})+\sum_{k=1}^{m} \Delta \tau_{i j}^{k}(\mathrm{t})
$$

In the formula above, $\Delta \tau_{i j}^{k}(t)$ is the pheromone concentration of $i \rightarrow j$ from time $t$ to $(t+1)$, in the most common model of ant cycle system:

$$
\Delta \tau_{i j}^{k}(\mathrm{t})=\left\{\begin{array}{l}
Q / L_{k}, \text { the ant pass the path } i \rightarrow j \\
0, \quad \text { else }
\end{array}\right.
$$

$\mathrm{Q}$ is constant $L_{k}$ is the price the ant pay in the cycle.

\subsection{The flow of ant colony algorithm}

Step1: Set nc $=0$ (nc is the iterations); $\tau_{i j}(0)=c$ (constant) $\Delta \tau_{i j}=0$ place $\mathrm{m}$ ants to $\mathrm{n}$ nodes; 2,

Step2:Place the starting positions of all ants to the current solution set $\mathrm{J}(\mathrm{i})$, move every ant $\mathrm{k}(\mathrm{k}=1$, 
$\ldots, \mathrm{m})$ to the next node $\mathrm{j}$ by proportion $p_{i j}^{k}$ and place $\mathrm{j}$ to $\mathrm{J}(\mathrm{i})$;

Step3: According to the update rules of pheromone, finding the shortest path after the ant $\mathrm{k}$ finish all the nodes and then update the pheromone concentration of every edge;

Step4: $\operatorname{Set} \Delta \tau_{i j}=0, n c=n c+1$.

Step5: If NC<the given ncmax, go to step2, or export the current best solution.

\subsection{The shortage of ant colony algorithm}

(1) Long searching time. The result will repeat between global optimal solution and local optimal solution.

(2) Premature convergence and easy stagnation. It doesn't get the global optimal solution until convergence, so the result is local optimal solution.

\section{Improved ant colony algorithm}

\subsection{Max-Min Ant System}

The Max-Min Ant System is put forward by Stutz T in 2009, it takes shape from the improvement of AS algorithm, and the main feature is that it only updates the pheromone of the optimal paths that the ant get through after every iteration, other ants are unrelated, and the pheromone concentration of every edge is strictly limited, it has a clear upper and lower bound. Although the opportunity of being chosen is small, but it reduces the possibility of stagnation and every path owns the same opportunity to be chosen. Thus, the new update rule of pheromone is as the following formula:

$$
\tau_{i j}(t+1)=\left[(1-\rho) \cdot \tau_{i j}(t)+\Delta \tau_{i j}^{\text {best }}\right]_{\tau_{\min }}^{\tau_{\max }}
$$

$\tau_{\max }$ And $\tau_{\min }$ are the upper and lower bound of pheromone concentration, the expression $[x]_{b}^{a}$ and $\Delta \tau_{i j}^{\text {best }}$ is defined as following:

$$
\begin{aligned}
& {[x]_{b}^{a}= \begin{cases}a & \text { if } x>\alpha \\
b & \text { if } x<b \\
x & \text { else }\end{cases} } \\
& \Delta \tau_{i j}^{\text {best }}=\left\{\begin{array}{l}
\frac{1}{L_{\text {best }}}, \text { if }(i, j) \text { is in the optimal path } \\
0, \text { else }
\end{array}\right.
\end{aligned}
$$

In the formula above, $L_{\text {best }}$ is the price of optimal path, $\tau_{\max }$ and $\tau_{\min }$ are obtained by experience and the concrete questions.

Thus, after the learning of object $O_{i}$, the probability the user $L_{0}$ choose $o_{j}$ as the next learning object is as following:

$$
P_{i j}^{L_{0}}(t)= \begin{cases}\frac{\left[\eta_{L_{0}, o_{j}}^{k-\text { level }}\right]^{\alpha_{1}} \times\left[\eta_{L_{0}, o_{j}}^{l-\text { ssyle }}\right]^{\alpha_{2}} \times\left[\tau_{i j}(t)\right]^{\beta}}{\sum\left(\left[\eta_{L_{0}, o_{j}}^{k-\text { level }}\right]^{\alpha_{1}} \times\left[\eta_{L_{0}, o_{j}}^{l-s y l e}\right]^{\alpha_{2}} \times\left[\tau_{i j}(t)\right]^{\beta}\right)}, & \left(o_{i} \rightarrow o_{j}\right) \in J(i) \\ 0, & \left(o_{i} \rightarrow o_{j}\right) \notin J(i)\end{cases}
$$

In the formula above, $\alpha_{1}$ and $\alpha_{2}$ are the influent parameters of the heuristic information of $\eta_{L_{o}, o_{j}}^{\text {k-level }}$ and $\eta_{L_{0}, o_{j}}^{l-s t l l} ; \mathrm{J}(\mathrm{i})$ is a possible learning path from $o_{i}$; if the user $L_{0}$ finish the learning of $o_{i}$ at time t, and the possibility he choose $\mathrm{J}(\mathrm{i})$ as the next learning path is as following:

$$
P_{J(i)}^{L_{0}}(t)=\prod_{J(\mathrm{i})} P_{i j}^{L_{0}}(t)
$$

\section{Example and analysis}

In order to verify the effectiveness and superiority of the improved algorithm, two comparative experiments are done. Experiment one makes use of the improved ant colony algorithm Experiment two makes use of the traditional ant colony algorithm which doesn't take the soldier's learning style into account. 
The match degree between the recommended result and the soldier's own preference can be calculated by the following formula:

$$
\operatorname{match}(R, P)=1-\frac{\sqrt{(\theta(a)-\gamma(a))^{2}+(\theta(b)-\gamma(b))^{2}+(\theta(c)-\gamma(c))^{2}}}{\sqrt{(\gamma(a))^{2}+(\gamma(b))^{2}+(\gamma(c))^{2}}}
$$

$\mathrm{R}$ is a set of the recommended result, $\mathrm{P}$ is a set of soldier's own preference $\theta(i)(i=a, b, c)$ is the recommended strength, $\gamma(j)(j=a, b, c)$ is the preference strength.

The comparative result is as following:

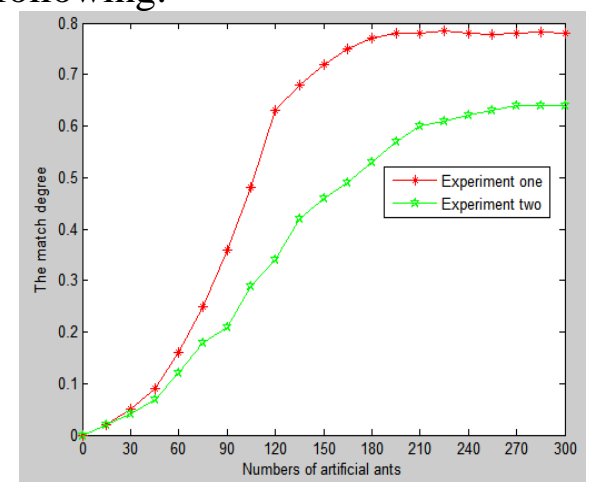

Figure.3 the comparative result of two experiments

From the figure, we can see that the improved ant colony algorithm can reach a higher precision only need about 200 ants, while the precision of traditional ant colony algorithm is improved to some extent with the increase of the numbers of ants, obviously, its convergent effect is not as good as that of the improved algorithm.

\section{Conclusion}

The paper puts forward to an improved ant colony algorithm aiming at improving the learning efficiency and professional skills of the soldiers of armored equipment support. After analyzing the shortage of the traditional algorithm, the paper not only makes use of adjacent users to guide the choose of path but also strictly limit the pheromone concentration of every edge and just update the pheromone of the optimal paths. The experiment result shows that the improved ant colony algorithm owns higher precision and the recommended learning path is almost coincide with the soldier's actual demand, this is a great help to the improvement of soldier's skills.

\section{References}

[1] Cheng Yan, 2011. A Method of Swarm Intelligence-Based Learning Path Recommendation for Online Learning. Journal of Systems \& Management, 20(2), p.232-237, Mar.

[2] Rongwei Gan, Qingshun Guo, Huiyou Chang, and Yang Yi, 2010. Improved ant colony optimization algorithm for the traveling salesman problems. Journal of Systems Engineering and Electronics Vol. 21, No. 2, April, p.329-333.

[3] Wang Yan-ling, Li Long-shu, Hu Zhe. 2008. Swarm Intelligence Optimization Algorithm. COM PUT ER TECHNOLOGY AND DEVELOPMENT, 18(8), pp. 114-117, Aug.

[4] Xiao Huimin, Ma Caijuan. 2013. Recommendation Method for Learning Path Based on Particle Swarm Optimization. HENAN SCIENCE, 31(12), pp.2190-2193, Dec.

[5] Xia Li-min, WANG Hua, DOU Qian, CHEN Ling. 2007. Research for optimal routing problem based on ant colony algorithm. Computer Engineering and Design, 28(16), pp.3957-3959, Aug.

[6] Yu Jian-ping, ZHOU Xin-min, CHEN Ming. 2010. Research on representative algorithms of swarm intelligence. Computer Engineering and Applications, 46(25), p.1-4.

[7] Zufeng Zhong, Hongyan Yang, 2012. Personalized Learning System Based on Data Mining for Experimental Platforms. National Conference on Information Technology and Computer Science (CITCS 2012):69-70.

[8] Zhong Keding, LIU Yang, 2012. The Design and Practice of Online Learning-guiding Strategies Based on Learning Style Theories. Open Education Research, 18(3), pp.83-88, Jun. 